\title{
COHERENT STATES OF PARA-BOSE OSCILLATORS
}

\author{
S. N. BISWAS and T. S. SANTHANAM
}

(Received 7 January 1980)

\begin{abstract}
Para-Bose coherent states, defined as "displaced" ground states, are obtained using the differential operator representation for the annihilation operator.
\end{abstract}

\section{Introduction}

Coherent states of the harmonic oscillator may be defined in many, but essentially equivalent, ways (see, for instance, [4]; generalized coherent states have been discussed in [9]). They can be defined as the eigenstates of the annihilation operator $a$,

$$
a|z>=z| z>,
$$

where $z$ is a complex number. The cohrent states can be defined equivalently as

$$
|z>=D(z)| 0>\text {, }
$$

where $\mid 0>$ denotes the vacuum which satisfies

$$
a \mid 0>=0 \text { and } N \mid 0>=0,
$$

the annihilation operator

$$
a=(q+i p) / \sqrt{ } 2,
$$

the number operator

$$
N=a^{+} a,
$$

and $D(z)$ is the displacement operator. These satisfy the commutation relations

$$
\left[a, a^{+}\right]=1
$$

and

$$
[a, N]=a \text {. }
$$


In order that $\mid z>$ are eigenstates of $a$, we have as a consequence of (1.2) and (1.3) that

$$
[a, D]=z D \text {. }
$$

Since $a \sim \partial / \partial a^{+}$(in view of (1.6a)), (1.7) gives the solution

$$
D=\exp \left(z a^{+}-z^{*} a\right),
$$

where the second term has been added to make $D$ unitary; $D$ is, in fact, an element of the Weyl group. Equations (1.1) and (1.7) are identical in content. Recently, Sharma, Mehta and Sudarshan [8] (SMS) have defined the coherent states of para-Bose oscillators as the eigenstates of the annihilation operator $A$. The para-Bose oscillators satisfy the equation of motion

$$
[A, N]=A,
$$

though they do not satisfy the canonical commutation relation (1.6a) [2]. For the para-Bose oscillators, the number operator $N$ is defined as

$$
N=\frac{1}{2}\left(A^{+} A+A A^{+}\right)-h_{0},
$$

where $h_{0}$ is the lowest eigenvalue of the Hamiltonian

$$
H=\frac{1}{2}\left(A^{+} A+A A^{+}\right) \text {. }
$$

The case $h_{0}=\frac{1}{2}$ corresponds to the standard oscillator. SMS have used the "Fock" representation of the annihilation operator $[3,6]$,

$$
(A)_{2 n, 2 n+1}=\left[2\left(n+h_{0}\right)\right]^{\frac{1}{2}}
$$

and

$$
(A)_{2 n-1,2 n}=(2 n)^{\frac{1}{2}},
$$

in the basis furnished by the eigenstates of $N$.

In this paper, we define the para-Bose coherent states as "displaced" ground states, that is, through (1.7). We make use of the differential operator representation for the annihilation operator $A$ in the Bargmann space of entire functions in the form obtained by Gray and Hurst [1]. Our result confirms that of SMS who have used the matrix representation of $A$ given by (1.12) and (1.13).

\section{Para-Bose oscillators}

Gray and Hurst [1] have obtained the following representation for $A$, as differential operators in the Bargmann space:

$$
A=\frac{d}{d z} \mp \frac{2 g_{1}}{z} P+\frac{1}{2 z}(1 \mp P) \text { and } A^{*}=z,
$$


where $p$, the order of the statistics, is given by

$$
p=4 g_{1}+2=2 h_{0},
$$

and the operator $P$ is given by

$$
P=\exp \left\{\pi i\left(z \frac{d}{d z}\right)\right\}
$$

The result of the operation of $A$ on any function $f(z)$ can be written down in the following matrix form :

$$
\left[\begin{array}{cc}
0 & \frac{d}{d z}+\frac{2 g_{1}+1}{z} \\
\frac{d}{d z}-\frac{2 g_{1}}{z} & 0
\end{array}\right]\left[\begin{array}{l}
f_{e} \\
f_{0}
\end{array}\right]
$$

and

$$
\left[\begin{array}{cc}
0 & \frac{d}{d z}-\frac{2 g_{1}}{z} \\
\frac{d}{d z}+\frac{2 g_{1}+1}{z} & 0
\end{array}\right]\left[\begin{array}{l}
f_{e} \\
f_{0}
\end{array}\right],
$$

for $g_{1}$ integral and half integral, respectively. In the above, $f_{e}$ and $f_{0}$ stand for the combinations $(f(z)+f(-z))$ and $(f(z)-f(-z))$, respectively. To see how this follows, one needs to make a substitution $z \rightarrow e^{\omega}$ in the original equation (2.1) and to note that the operator $P$ simply becomes a translation operator in $\omega$-space. In a similar manner, the operation of $A^{+}$on $f$ reduces to the form

$$
\left[\begin{array}{ll}
0 & z \\
z & 0
\end{array}\right]\left[\begin{array}{l}
f_{e} \\
f_{0}
\end{array}\right]
$$

The corresponding number operator $N$ can be written as

$$
N=\frac{1}{2}\left(z \frac{d}{d z}+1\right)
$$

However, as is clear from (2.2), these yield only the representations for even order. For the case where $p$ is odd, one finds

$$
A=\left[\begin{array}{lr}
0 & \frac{d}{d z} \mp \frac{2 g_{1}}{z} \\
\frac{d}{d z} \mp \frac{2 g_{1}}{z} & 0
\end{array}\right] \text { and } A^{+}=z,
$$

where now

$$
p=4 g_{1}+1=2 h_{0}
$$


Here the upper and lower signs refer to $g_{1}$ integral and half-integral, respectively.

\section{Para-Bose coherent states}

We define para-Bose coherent states as

$$
|z>=D(\lambda z)| 0>
$$

where the operator $D$ satisfies

$$
[A, D]=\lambda D \text {. }
$$

In order to solve (3.2) we take the matrix elements

$$
\left\langle z|A D-D A| \psi_{0}\right\rangle=\lambda\left\langle z|D| \psi_{0}\right\rangle,
$$

where $\left\langle z \mid \psi_{0}\right\rangle$ stands for the projection of a state $\psi_{0}$ into Bargmann space. The operator $A$ has the form of the differential operator discussed in the earlier section in the space of functions $\left\langle z \mid \psi_{0}\right\rangle$. To solve (3.3) we make the following ansat $z$, namely, we choose the state $\psi_{0}$ such that

$$
\left\langle z|A| \psi_{0}\right\rangle=0
$$

in this case the equation for $D$ turns out to be

$$
\left\langle z|A D| \psi_{0}\right\rangle=\lambda\left\langle z|D| \psi_{0}\right\rangle
$$

This requirement at $\lambda=0$, by Schur's lemma, is a way of ensuring that the representation of the canonical commutation relations carrying the parabose operators is irreducible. Writing $f(z)$ for $\left\langle z|D| \psi_{0}\right\rangle$ we solve (3.5) in the following four different cases.

(i) Para-Bose order $p$ is even and $g_{1}$ is integral. From (2.4) we have the coupled equations for $f(z)$,

$$
\left(\frac{d}{d z}+\frac{2 g_{1}+1}{z}\right) f_{0}=\lambda f_{e}
$$

and

$$
\left(\frac{d}{d z}-\frac{2 g_{1}}{z}\right) f_{e}=\lambda f_{0}
$$

These equations can be combined to yield

$$
f_{0}^{\prime \prime}+\frac{1}{z} f_{0}^{\prime}-\frac{\left(2 g_{1}+1\right)^{2}}{z^{2}} f_{0}=\lambda^{2} f_{0}
$$


and

$$
f_{e}^{\prime \prime}+\frac{1}{z} f_{e}^{\prime \prime}-\frac{\left(2 g_{1}\right)^{2}}{z^{2}} f_{e}=\lambda^{2} f_{e}
$$

The acceptable solutions, which are not singular at the origin, of (3.8) and (3.9) are, respectively,

$$
f_{0}(z)=I_{2 g_{1}+1}(\lambda z)=I_{h_{0}}(\lambda z)
$$

and

$$
f_{e}(z)=I_{2 g_{1}}(\lambda z)=I_{h_{0}-1}(\lambda z)
$$

apart from multiplicative constants which we fix by requiring that, at $\lambda=0$, the operator reduces to the identity. Here $I_{v}$ is the modified Bessel function of the $v$ th order [5]. The particular solution $\left\langle z \mid \psi_{0}\right\rangle$ satisfies the homogeneous equations

$$
\left(\frac{d}{d z}+\frac{2 g_{1}+1}{z}\right)\left\langle z \mid \psi_{0}\right\rangle_{\text {even }}=0
$$

and

$$
\left(\frac{d}{d z}-\frac{2 g_{1}}{z}\right)\left\langle z \mid \psi_{0}\right\rangle_{\text {odd }}=0 .
$$

Equation (3.12) gives $\left\langle z \mid \psi_{0}\right\rangle_{\text {even }} \sim z^{-\left(2 g_{1}+1\right)}$ which is singular at $z=0$ and is thus unphysical; on the other hand, (3.13) gives a regular solution, namely,

$$
\left\langle z \mid \psi_{0}\right\rangle=\left\langle z \mid \psi_{0}\right\rangle_{\text {odd }} \sim z^{2 g_{1}}=z^{h_{0}-1} .
$$

Thus we find from the solutions of (3.8), (3.9) and (3.14) that $D$ is given by

$$
\begin{aligned}
\left\langle z|D| \psi_{0}\right\rangle & =\left\{\left\langle z \mid \psi_{0}\right\rangle\right\}^{-1}\left(f_{e}+f_{0}\right) \\
& =z^{1-h_{0}}\left\{I_{h_{0}}+I_{h_{0}-1}\right\},
\end{aligned}
$$

apart from a multiplicative constant.

(ii) peven, $g_{1}$ half integral. It is obvious from (2.5) that the solution of $D$ is the same as that given by (3.15).

(iii) $p$ odd, $g_{1}$ integral. From (2.8) we now have the coupled equations

$$
\left(\frac{d}{d z}+\frac{2 g_{1}}{z}\right) f_{e}=\lambda f_{0}
$$

and

$$
\left(\frac{d}{d z}-\frac{2 g_{1}}{z}\right) f_{0}=\lambda f_{e}
$$

Following the procedure as above we have the solutions 


$$
\begin{aligned}
f_{0} & =(\lambda z)^{\frac{1}{2}} I_{2 g_{1}+t}(\lambda z) \\
& =(\lambda z)^{\frac{1}{2}} I_{h_{0}}(\lambda z)
\end{aligned}
$$

and

$$
\begin{aligned}
f_{e} & =(\lambda z)^{\frac{1}{2}} I_{2 g_{1}-\frac{1}{2}}(\lambda z) \\
& =(\lambda z)^{\frac{1}{2}} I_{h_{0}-1}(\lambda z) .
\end{aligned}
$$

The particular solutions $\left\langle z \mid \psi_{0}\right\rangle$ in this case turn out to be

$$
\left\langle z \mid \psi_{0}\right\rangle=z^{ \pm 2 g_{1}}
$$

Keeping the regular solution, as before,

$$
\left\langle z \mid \psi_{0}\right\rangle=z^{2 g_{1}},
$$

we find from (3.18), (3.19) and (3.24) that

$$
\begin{aligned}
\left\langle z|D| \psi_{0}\right\rangle & =z^{-2 g_{1}+\frac{1}{2}}\left\{I_{h_{0}}+I_{h_{0}-1}\right\} \\
& =z^{\left(1-h_{0}\right)}\left\{I_{h_{0}}+I_{h_{0}-1}\right\},
\end{aligned}
$$

which is the same as (3.15).

(iv) $p$ odd, $g_{1}$ half integral. As before, the solution for this case is the same as given by (3.15).

Thus, in all these cases we get the unique solution and it is given by

$$
\left\langle z|D| \psi_{0}\right\rangle=(\lambda z)^{\left(1-h_{0}\right)}\left\{I_{h_{0}}(\lambda z)+I_{h_{0}-1}(\lambda z)\right\} .
$$

As mentioned, we require that $D=1$ for $\lambda=0$; we multiply (3.23) by the constant factor $\Gamma\left(h_{0}\right)(\lambda / 2)^{1-h_{0}}$ so that the required solution becomes

$$
\begin{aligned}
\left\langle z|D| \psi_{0}\right\rangle & =\Gamma\left(h_{0}\right)\left(\frac{\lambda z}{2}\right)^{1-h_{0}}\left\{I_{h_{0}}(\lambda z)+I_{h_{0}-1}(\lambda z)\right\} \\
& =\sum_{n=0}^{\infty} \frac{\Gamma\left(h_{0}\right)}{\Gamma\left[\frac{1}{2} n\right]+1 \Gamma\left[\frac{1}{2}(n+1)\right]+h_{0}}\left(\frac{\lambda z}{2}\right)^{n},
\end{aligned}
$$

where the square brackets stand for the integral part. This is exactly the solution given by SMS. This is not surprising since the representation is irreducible and, by the well-known theorem of Rellich and von Neumann [7], all such representations are isomorphic. The para-Bose coherent state $|\lambda\rangle$, corresponding to the eigenvalue $\lambda$, thus becomes

$$
|\lambda\rangle=D\left(\lambda A^{+}\right)|0\rangle
$$

where we have replaced $z$ by the operator $A^{+}$.

We have now to choose the normalization $\mu$ so that 


$$
\mu^{2}\langle\lambda \mid \lambda\rangle=\mu^{2}\left\langle 0\left|D^{+}\left(\lambda^{*} A\right) D\left(\lambda A^{+}\right)\right| 0\right\rangle=1 .
$$

To evaluate (3.26) we follow SMS and make use of the following commutation relations which follow simply from the equation of motion (1.9):

$$
\left[A, A^{+2 k}\right]=2 \mathrm{kA}^{+2 k-1}
$$

and

$$
\left[A, A^{+2 k+1}\right]=A^{+2 k}\left\{2 k+\left[A, A^{+}\right]\right\},
$$

where we use the result that the commutator $\left[A, A^{+}\right]$commutes with $A^{2 k}$ and $A^{+2 k}$. Using (3.27), it follows that

$$
\langle 0|\left\langle A^{m} A^{+m} \mid 0\right\rangle=2^{m} \frac{\Gamma\left\{\left[\frac{1}{2} m\right]+1\right\} \Gamma\left\{\left[\frac{1}{2}(m+1)\right]+h_{0}\right\}}{\Gamma\left\{h_{0}\right\}},
$$

and thus

$$
\mu=\left\{D\left\langle|\lambda|^{2}\right\rangle\right\}^{-\frac{1}{2}}
$$

The properly normalized para-Bose coherent state thus becomes

$$
|\lambda\rangle=\left\{D\left(|\lambda|^{2}\right)\right\}^{-\frac{1}{2}} D\left(\lambda A^{+}\right)|0\rangle \text {. }
$$

It is easy to see that, when $h_{0}=\frac{1}{2}$ (normal oscillator),

$$
D\left(|\lambda|^{2}\right)=\exp |\lambda|^{2}
$$

as has already been pointed out by SMS. The important point, however, is that, for para-Bose oscillators, $D\left(\lambda A^{+}\right)$does not form a group.

\section{Conclusions}

We have outlined an alternate method of obtaining para-Bose coherent states, defined as "displaced" ground states, using the differential operator representation of the annihilation operator in Bargmann space derived by Gray and Hurst [1]. Our result agrees with that of Sharma, Mehta and Sudarshan [8] who have used the "Fock" representation.

We must mention that the solution for $D$ obtained here is a particular one; in general, there are other solutions of (3.3). These, by virtue of the Rellich-von Neumann theorem, should all be isomorphic, and therefore carry the same physical meaning.

That the displacement operator will be a modified Bessel function is clear from the form of the annihilation operator given in (2.1).

The usefulness of the differential operator representation has been discussed by Klauder and Sudarshan [4], page 127, for the coherent states of the oscillator. The main point is that one can discuss many important consequences of the coherent 
states most easily from the analytic properties of the representations in Bargmann space. In particular, the differential equation satisfied by the evolution operator directly leads to a solution in the normal ordered form. The completeness (actually, overcompleteness) property of the coherent states as well as their non-orthogonal nature are most easily exhibited in Bargmann space.

It is, however, futile at the present stage to discuss in more detail the usefulness of the representations in Bargmann space, especially since the concept of para-Bose coherent states is nascent and yet to find its roots in physical problems.

\section{Acknowledgement}

We are grateful to Professor K. J. Le Couteur for his kind hospitality and keen interest in the problem.

\section{References}

[1] D. A. Gray and C. A. Hurst, "Explicit representations of a single parabose operator", J. Math. Phys. 16 (1975), 326-333.

[2] H. S. Green, “A generalized method of field quantization", Phys. Rev. 90 (1953), 270-273.

[3] T. F. Jordan, N. Mukunda and S. V. Pepper, "Irreducible representations of generalized oscillator operators", J. Math. Phys. 4 (1963), 1089-1096.

[4] J. R. Klauder and E. C. G. Sudarshan, Fundamentals of quantum optics (Benjamin, 1968).

[5] N. N. Lebeder, Special functions and their application (Prentice-Hall, 1965), p. 108.

[6] L. O'Raifeartaigh and C. Ryan, Proc. Roy. Irish. Acad. A. 62 (1963), 93.

[7] F. Rellich, "Der Eindeutigkeitssatz für die Läsungen der quantummechanischen Vertanschumgsrelationen", Nachr. Akad. Wiss. Göttingen, Math. Physik. K 1 (1946), 107-115.

[8] J. K. Sharma, C. L. Mehta and E. C. G. Sudarshan, "Para-Bose coherent states", J. Math. Phys. 19 (1978), 2089-2093.

[9] T. S. Santhanam, Invited talk at the Albert Einstein centennial week symposium on Symmetries in science held in Carbondale, Southern Illinois, U.S.A., 28 February to 2 March 1979. To appear in the Proceedings (Plenum Press).

Department of Theoretical Physics

Research School of Physical Sciences

Australian National University

Canberra

A.C.T. 2600 